\title{
Humanitarian Device Exemption
}

National Cancer Institute

\section{Source}

National Cancer Institute. Humanitarian Device Exemption. NCI Thesaurus. Code C80440.

A premarket submission to FDA for a device intended to benefit patients by treating or diagnosing a condition affecting fewer than 4,000 individuals in the United States per year. An HDE is similar in form and content to a premarket approval (PMA) but is exempt from the effectiveness requirements of a PMA. 